19 Revue d'histoire du XIXe siècle

Société d'histoire de la révolution de 1848 et des

révolutions du XIXe siècle

22 | 2001

Autour de Décembre 1851

\title{
Document : le Coup d'Etat vu par un ouvrier
}

\section{Paul Chauvet}

URL : http://journals.openedition.org/rh19/261

DOI : $10.4000 /$ rh 19.261

ISSN : $1777-5329$

Éditeur

La Société de 1848

Édition imprimée

Date de publication : 1 juin 2001

ISSN : 1265-1354

\section{Référence électronique}

Paul Chauvet, " Document : le Coup d'Etat vu par un ouvrier », Revue d'histoire du XIXe siècle [En ligne], 22 | 2001, mis en ligne le 27 juin 2005, consulté le 21 avril 2019. URL : http://journals.openedition.org/ rh19/261 ; DOl : 10.4000/rh19.261

Ce document a été généré automatiquement le 21 avril 2019

Tous droits réservés 


\title{
Document : le Coup d'Etat vu par un ouvrier
}

\author{
Paul Chauvet
}

1 On a beaucoup reproché a la classe ouvrière sa passivité au cours des événements de décembre 1851 sans, trop souvent, se donner la peine d'examiner quelles pouvaient être les raisons qui étaient à l'origine de cette attitude.

2 Sans nier l'effet de la propagande bonapartiste sur la partie la moins consciente $d u$ prolétariat, il semble bien qu'on puisse attribuer cette indifférence aux déceptions de toutes sortes qui vinrent accabler les ouvriers dès le lendemain de la révolution de février 1848.

3 Le chômage, voulu et organisé par la bourgeoisie inquiète et mécontente, les massacres de juin, la répression qui suivit et qui continua à s'abattre sur les meilleurs militants, ainsi que le manque de fermeté des députés de la Montagne, avaient profondément découragé, on pourrait dire ulcéré, les ouvriers qui avaient vu s'évanouir tous les espoirs qu'avait suscité la chute de la royauté constitutionnelle. De plus, ce qu'on oublie trop, il y avait longtemps, en décembre 1851, que les armes qui leur avaient été données après février 1848 leur avaient été enlevées.

4 Dans ces conditions, qu'auraient-ils pu faire, sans armes, sans chefs vraiment capables? Se faire tuer pour une bourgeoisie qui, le danger passé, les aurait de nouveau trahis? La grande majorité des ouvriers estimèrent que le jeu n'en valait pas la chandelle et ils restèrent chez eux.

5 À cette époque, l'aile avancée de la classe ouvrière parisienne était incontestablement représentée par la typographie, dont l'un des meilleurs militants, Simon Parmentier avait été, en mars 1848, président de ce Comité Central des Ouvriers du Département de la Seine, dont l'action politique avait été alors fort importante.

6 Parmi les militants qui donnaient alors a la typographie parisienne un éclat tout particulier, se trouvait un compositeur nommé Bosson, lequel, bien que n'ayant pas fait le 
coup de feu, se trouva pris dans le vaste coup de filet qui fut alors jeté sur le Paris ouvrier et républicain.

7 Bosson avait évidemment un passé plutôt chargé. À l'âge de 11 ans, il avait, en juillet 1830, participé a la prise du Louvre. Il avait fait le coup de feu en février et en juin 1848, et avait échappé de peu a la transportation. Peu de temps avant le coup d'État, il avait assumé pendant plusieurs mois la présidence de la Société Typographique parisienne qui passait - avec juste raison - pour être une citadelle républicaine et socialiste.

8 En 1896, Besson alors âgé de 77 ans entreprit de faire le récit des événements auxquels il avait été mêlé dans sa jeunesse. Ces notes manuscrites sont conservées a la Bibliothèque des Arts Graphiques. Toutes réserves faites pour quelques inexactitudes qui ne sont peutêtre que des défaillances de mémoire, elles constituent une documentation particulièrement intéressante, notamment en ce qui concerne l'attitude du corps typographique parisien sous la deuxième république et en 1851.

9 Les pages qui suivent, consacrées au coup de force de Louis Napoléon, apportent d'intéressantes précisions sur les réactions parisiennes, spécialement de la typographie, tout en montrant l'aveuglement de certains hommes politiques de gauche, lesquels comme Thiers, affectaient de considérer Louis Napoléon comme un demi-imbécile. P.C.

11 contre la loi dite des 21 , dont Thiers faisait le plus ornement, cette loi qui, sons prétexte de réglementer le suffrage universel, avait supprimé trois millions d'électeurs, c'est-àdire la partie la plus active de la nation. Girardin avait déclaré qu'il se ferait tuer sur sa chaise curule plutôt que d'en descendre.

13 Aussi la Typographie, fidèle à son amour de la liberté, pensa offrir la présidence du banquet de 1851 à Émile de Girardin, l'homme de la Liberté. Je fus délégué avec deux membres du Comité pour lui offrir la présidence. Après l'avoir félicité de sa brochure qui avait déterminé notre offre, Girardin nous dit: “Depuis que j'ai eu ce malheureux duel avec Armand Carrel, je me suis promis de ne jamais assister à une réunion politique ". On lui fit observer que notre banquet n'était que corporatif il ne voulut rien entendre: "Je ne veux pas me produire en public". Il nous fit don de 200 exemplaires de sa brochure. Il ajouta: "Puisque vous avez en confiance en moi, je vais vous faire une confidence : le coup d'État est décidé; si ce n'est pas dans huit jours, ce sera dans quinze, dans un mois, deux mois, trois mois, six, mais suivant le moment opportun ". Depuis ce mot m'est toujours resté dans la tête, car tandis que l'Italien Gambetta avec ses amis nous ont toujours lanterné avec le moment opportun pendant vingt-six ans, le bandit le choisit le 2 décembre, environ six mois après notre visite à Girardin qui eut lieu en juillet. Pour moi, j'étais bien convaincu que l'homme qui avait fait Boulogne et Strasbourg ne s'arrêterait pas en si beau chemin, car la cuisinière de Duverger, deux fois directeur de l'Imprimerie Nationale, qui avait élevé Daru fils m'avait dit que Verhuel avait fait venir à l'Élysée Daru pour lui dire que son nom appartenait à l'Empire et qu'il devait marcher avec. Daru lui avoua qu'il ne pouvait rompre des amitiés avec les d'Orléans. Verhuel riposta: “Vous avez tort: je suis sur les marches du trône je me ferais sabrer plutôt que d'en descendre!" 
14 La Typographie étant prévenue, je me fis un devoir d'aller répandre la nouvelle pour se tenir en garde Le premier que je vis fut Pierre Leroux, auquel je fis part de la confidence. Il y avait là Pauline Roland et le Docteur François. Pierre Leroux fit un sourire d'incrédulité, il me dit: “Je connais mon petit Louis : il en est incapable !" Pauline Roland qui était une frêle créature, un souffle, bondit comme un cabri : "Votre petit Louis !..., Mais j'aime cent mille fois mieux le boucher Cavaignac que votre petit Louis : c'est une vipère ! ..." Elle avait le pressentiment de ce qui devait lui arriver. L'Empire eut peur d'une femme si délicate : elle fut transportée à Lambessa où, pendant le trajet, les femmes arabes, émues de compassion, la mettaient sur un âne ; elle fut frappée d'insolation. Quand on vit qu'elle était frappée à mort, on lui accorda sa grâce. Elle ne put aller que jusqu'à Lyon, où son fils Jean arriva pour embrasser le cadavre encore chaud de sa mère. Quant au Docteur François, il ne dit pas un mot, et je m'en allai le cœur serré. Je vis ensuite mon ami Baudin qui eut également un sourire d'incrédulité, avec un très léger haussement d'épaule, il me dit : “Je serai là !" Oh ! en effet, il se trouva là pour aller au temple de la gloire et se faire tuer en protestant. J'étais atterré de voir que les deux hommes sur lesquels je comptais le plus pour déjouer les projets du criminel, n'y croyaient pas. Obstiné comme un Breton, je prévins la Montagne (pauvre Montagne!) rue du Hasard!... et plus de 3000 citoyens. Il Semblait que Baudin eût donné le mot. Tous répondaient : "Nous serons là ! " - " Il est trop bête!" - “Il n'oserait pas!" ajoutaient-ils. J'avais beau invoquer Strasbourg et Boulogne, la confiance d'enfant des Gaulois avait le dessus: on me disait qu'on me conduirait à Charenton et lui à Vincennes : Plût aux dieux comme disait Socrate, que cela fût arrivé ! Mais les caricatures, les plaisanteries gauloises qui affirmaient qu'il ne savait dire que: “Très flatté !" tout faisait passer le taciturne hollandais pour idiot. Le journal Le Napoléon endormait les parisiens en écrivant huit jours avant le crime : "Bourgeois de Paris, dormez tranquilles, il n'y aura jamais de coup d'État ".

15 J'avais accompli mon devoir mais le tour fut joué pour n'avoir pas su prévoir. Tout le monde s'exclamait: “ Nous ne l'aurions jamais cru!" La Typographie réunie voulait faire sortir le drapeau : c'était aller au devant d'un massacre : Je dis "Allons voir les étudiants : nous verrons de quels sentiments ils sont animés". On leur porta des petits papiers imprimés clandestinement, portant que le général Neumayer avec sa division marchait de Strasbourg sur Paris pour défendre la République. On voulut les entraîner, mais ils se contentèrent de nous dire: “Dépêchez-vous de vous sauver : voilà les sergents de ville armés de fusils qui débouchent par la rue Hautefeuille!" C'était là tout leur concours. Cela jeta un froid sur l'élan et l'idée fut abandonnée. Le massacre des boulevard eut lieu : 1800 vieillards, femmes et enfants furent tués. “ C'était une révolution militaire ", comme a dit Granier dit de Cassagnac. L'armée offrait le combat à une population qui avait été désarmée trois mois auparavant par le préfet de police Carlier. Pour donner une idée de l'état d'âme de la soldatesque, je rentrais chez moi dans la Cité, lorsque je vis le Pont Saint-Michel barré par une compagnie de gendarmes d'élite qui avaient allumé les feux de bivouac. Je m'adressai au capitaine pour me laisser passer. L'aimable guerrier me répondit : "Venez donc chercher nos armes!" Il offrait le combat à un homme désarmé. J'avais une telle rage au cœur que si j'avais eu un pistolet, je lui aurais brûlé la gueule. Je dus aller me coucher sur des rognures à l'imprimerie.

16 Du reste, en votant la loi électorale, l'Assemblée avait commis un crime contre le suffrage universel et fourni au Hollandais un puissant atout pour perpétrer son attentat : en effet, en restituant le suffrage universel à tous, le peuple fut satisfait et ne se révolta pas, et quand les députés, après avoir vu les portes de l'Assemblée fermées, se réunirent à la 
mairie du $10^{\mathrm{e}}$, rue de Grenelle alors, présidés par le général Oudinot, l'écraseur de la République romaine, protégés par un bataillon de la garde nationale et un bataillon de chasseurs à pied, et après que le général Changarnier eut solennellement dit: “Législateurs, délibérez en paix !" et qu'ils furent par ordre, arrêtés par les chasseurs et conduits à la caserne du quai d'Orsay, le peuple applaudissait à outrance partout où ils passaient. Ah ! comme mon ami Lagrange, de Lyon, avait raison quand, avant le vote de cette abominable loi, il dit à Garnier-Pagès et à Crémieux demandant ce qu'il fallait faire : "Il faut descendre dans la rue et faire un appel aux armes! " Ils baissèrent la tête, alors, les prenant par les épaules et les poussant vers la salle des séances : "Allez donc voter !". Oh! que les députés furent coupables! Avec Thiers en tête, ils furent mille fois plus indignes que la vipère (Louis-Napoléon).

17 Petet m'avait prévenu qu'un mandat d'arrestation étant lancé contre les 15 membres du Comité et les 52 délégués des corporations : c'était sur la dénonciation de trois maîtres imprimeurs au nom de leur Chambre. Mon patron était un des trois. Les perquisitions furent faites; Paquai fut renvoyé en Savoie; le plus jeune des membres du Comité chez lequel on avait trouvé un mauvais pistolet, fut, seul, arrêté ; Wahry, délégué des tailleurs et disciple de Pierre Leroux, fut reconduit en Suisse. Plusieurs délégués furent arrêtés, relaxés, repris et envoyés à Cayenne. Depuis plus de six mois, Viguier, le benjamin de Pierre Leroux, et moi, nous ne faisions plus partie du Comité; cependant, chose très bizarre, on nous arrêta.

On fit fermer le comptoir ${ }^{1}$.

19 De même, on détruisit l'association solidaire des 14 Corporations de Rouen, dirigée par le cordonnier Chevrelle, disciple de Pierre Leroux. Il est mort il y a cinq ans. C'était un homme très sévère pour lui, très indulgent pour les autres ; d'un esprit très droit, il faisait des observations toujours justes, il savait encourager et exciter les efforts de chacun: C'était merveille de voir l'entrain qui existait dans l'association; tous étaient heureux et se surpassaient ; les travaux étaient bien faits ; aussi la maison était très prospère, car les Rouennais s'arrachaient les marchandise et les bourgeois étaient en rage contre ces solidaires qu'ils dénonçaient chaque jour au Préfet, lequel, lors du coup d'État, le fit venir nuitamment dans son cabinet et, lui prenant la main, dit: "Je n'ai que des félicitations et des louanges à vous faire, mais partez de suite, parce que les ordres sont impitoyables; si les bourgeois vous voyaient, ils me forceraient à vous arrêter et vous seriez envoyé à Cayenne!" Mon pauvre ami ne se le fit pas dire deux fois : il partit de suite; mais quel crève-cœur pour lui quand il songeait à ce qu'il était forcé d'abandonner. Tandis que Pierre Leroux, par suite de la révolution de février, avait vu l'Association de Boussac se dissoudre faute de travail et être obligé de rembourser les dettes, l'Association rouennaise - fondée par Beaufour, publiciste normand, un de ses disciples - composée de cuisiniers, chapeliers, cordonniers et tailleurs se maintenait péniblement parce que le directeur n'était pas un homme pratique ; c'est alors qu'il offrît à son pays Chevrelle d'en prendre la direction; notre ami ne voulut pas : ce n'est qu'à force de supplications de Viguier et de moi qu'il se décida à accepter. Aussi, en voyant détruire son œuvre si prospère qu'il avait eu tant de peine à organiser, on peut juger du désespoir profond qu'il éprouva. Depuis, chaque fois que je le rencontrais, en me donnant la main, il me disait: " Oh! les infâmes bourgeois !..."

20 Pendant que Viguier et moi avions pris la place dans la place [sic] qu'occupait son ami intime Cabet, qui était sorti la veille pour aller mourir en exil, je me demandais comment il se faisait que mon compagnon si calme, si effacé, avait pu mériter une arrestation dans 
un moment si terrible, lui qui venait de se marier nouvellement; passe pour moi : j'avais de très mauvaises notes et j'avais été condamné à la transportation ; de plus, je ne pouvais pas retenir ma langue; mais lui si tranquille, comment avait-il pu concourir cette rigueur? Après avoir réfléchi profondément, j'eus la conviction que nous étions étroitement surveillés ; que pas une démarche, un fait n'étaient ignorés. On avait constaté que c'était lui la cheville ouvrière de ce grand mouvement de marche en avant. Élève de l'École Normale, il possédait une activité incroyable appuyée par le savoir: comme secrétaire, tous ses procès-verbaux très clairs, étaient tenus à jour et toutes les questions graves à poser au Comité étaient élucidées à l'avance avec la méthode de notre confrère Franklin, de sorte que toutes les difficultés et tous les avantages étaient signalés ; alors le Comité pouvait toujours juger en connaissance de cause; ce fut lui qui, pour éviter les mises-bas, me fit courir chez tous les maîtres pour faire de la conciliation et je fus surpris des bons résultats de ces démarches. L'ordre du jour des séances était toujours réglé à l'avance : facilité et pas de temps perdu. Comme on voit, c'était un grand criminel!

21 Nous étions accusés de violenter les patrons et d'être maîtres chez eux, et d'être à la tête d'une société prête à jeter 1800 hommes dans les barricades. Deux jours de suite, je fus à l'interrogatoire pendant quatre heures. L'interrogateur Brault me fit mettre près de la cheminée et fit un feu d'enfer je cuisais : c'était un reste d'inquisition. Je n'eus pas de peine à démontrer que jamais nous n'avions procédé par la violence, que nous avions toujours respecté la liberté du travail et que pour prouver les sagesses de nos réclamations, sur 22 affaires jugées par la Conférence, en moyenne, nous en avions gagnées 21 ; quant à la Société, nous n'avions aucune action politique puisque tous les partis étaient représentés dans cette agglomération corporative. Le confrère qui avait donné les renseignements n'avait pas volé son argent. J'indiquai à Viguier plusieurs points à défendre. Enfin à nous deux, nous avions plaidé pendant douze heures pour défendre la Société. M. Brault nous fit venir et nous dit: "En temps ordinaire je vous aurais mis immédiatement en liberté ; mais en en moment sous la puissance des conseils de guerre, je ferai tout mon possible, mais je ne vous promets rien ". Quelques jours après nous étions libres. Petet nous avait recommandés au Directeur du Dépôt qui nous dit: “Avant de quitter la Préfecture, vous ferez bien d'aller remercier M. Tripier-Lefranc, secrétaire général, et $M$. de Paris, chef de la Division des Sociétés, qui ont été très bienveillants pour vous". Après avoir remercié $M$. Tripier-Lefranc qui nous promit son appui pour la Société, nous avons également rendu visite à M. de Paris, un charmant homme qui nous conseilla de régulariser la Société en acceptant le fait accompli ; par contrepartie, le Préfet reconnaîtrait la Société telle qu'elle était constituée. C'eût été une bonne affaire que nous aurions acceptée de grand cœur, mais nous n'étions plus du Comité et ne pouvions rien. En sortant, quelqu'un me frappa sur l'épaule: “Vous êtes le fils du père Victor? - Oui, monsieur. - J'ai beaucoup connu votre père qui était mon ami. J'ai un conseil à vous donner : vous avez un dossier très chargé ; dépêchez-vous de vous éloigner, car vous pourriez être repris et envoyé à Cayenne. Dans quelques mois le calme sera fait, vous pourrez alors revenir". Je le remerciai beaucoup et je pris de suite un passeport pour la Suisse pour éviter ce qui m'était déjà arrivé. Avant de partir, j'engageai la "Mère" ${ }^{2}$ à régulariser la position. Mais Parmentier, Antoine Viez et compagnie voulurent jouer à la Société secrète et mes amis, se drapant dans leur dignité : “Au nom de la Constitution et des droits qu'elle consacre, etc. ", ne voulurent rien reconnaitre. À quoi M. de Paris leur répondit: “Mes enfants, la Constitution n'existe plus; un ordre de choses nouveau est établi ; en ne l'acceptant pas, le jour où il plaira au Préfet de dissoudre 
votre Société, vous serez désarmés, tandis que si vous acceptez, tous vos droits seraient consacrés ". Nos amis furent inflexibles. Une première atteinte fut portée.

22

23 Louis Napoléon, il fit partie un moment de ces équipes d'ouvriers qui essayèrent, vers 1861-1862, de concilier l'Empire et les classes laborieuses.

24

P. C.

\section{NOTES}

1. Ce "Comptoir" consistait en une sorte de magasin qui fonctionnait sous le contrôle de la Société Typographique parisienne. - P. C.

2. La typographie parisienne était alors divisée entre deux groupes rivaux : la Mère, qui n'était autre que l'ancienne Société Typographique, et la Fille. Cette division avait surtout été provoquée par des questions de mutualisme. - P.C. 